\title{
Chromatographic Separation and Characterization of Capsaicinoids and Their Analogues*
}

\author{
Junlian Wang ${ }^{1}$, Li Zhou ${ }^{2}$, Lei Wang ${ }^{2}$, Zhenghong Peng ${ }^{3}$, Shengze Zhou ${ }^{4}$, Xiangfeng Zhou ${ }^{5}$, \\ Xiaobin Zhang ${ }^{1}$, Bixian Peng ${ }^{6 \#}$
}

${ }^{1}$ Department of Materials Science and Engineering, Zhejiang University, Hangzhou, China; ${ }^{2}$ Chenguang Engineering Technology Research Center of Natural Pigments, Hebei, China; ${ }^{3}$ Beijing TianGongLongYuan International Biochemical Company, Beijing, China; ${ }^{4}$ Beijing JiuZhouLongLing Environmental Protection Material Company, Beijing, China; ${ }^{5}$ Institute of Chemistry, Chinese Academy of Sciences (CAS), Beijing, China; ${ }^{6}$ Technical Institute of Physics and Chemistry, CAS, Beijing, China.

Email: "\#pengbx@mail.ipc.ac.cn

Received December $19^{\text {th }}, 2010$; revised May $4^{\text {th }}, 2011$; accepted May $15^{\text {th }}, 2011$.

\begin{abstract}
An attempt was made to establish a chromatographic separation and analysis method for capsaicinoids and their analogues. A series of factors,such as choice of column and eluents, eluent composition,flow rate of eluent and operational temperature were correlated, countereacted and optimized to attain appropriate separation efficiency and finalize the experimentally oprational procedures as a whole, permiting a mixture comprising 8 capsaicinoids including analogues to be separated and Characterized with an allowed deviations and within a period of 36 minutes via 1 test as well.
\end{abstract}

\section{Keywords: Capsaicinoid, Chromatography, Separation, Characterization}

\section{Introduction}

In our previous paper [1], it has been introduced that capsaicin had been effectuated to have shown mutagenic, anti-proliferative and anticarcinogenic properties toward human prostate gland's, lung cancer's and leukemic cells [2-10] and highlighted that capsaicinoids differ from each other in pungency depending on the molecular concrete structure involved, leading to a conclusion that capsaicin and dihydrocapsaicin had been ranked to be the most pungent ones. Combining the research results originated and come from completely different 2 sectorsone is the curative effect of capsaicin displayed in pharmacology with respect to different cancer cells, the other is pungency structure dependence exploited in bioorganic chemistry-as a whole, it seems to us to have seen what a bit light of early dawn and we are hopefully imagined that the potential biologically curative value and even the possible clinical administration and observation of capsaicinoid deserve attention and concerns of

\footnotetext{
${ }^{*}$ Capsaicinoids are referred to all the pungent members of capsaicin family present in natural hot pepper. Analogues are referred to those artificial pungent compounds whose molecular structures are similar to capsaicinoids but unable to be detected in native hot pepper.
}

medical researchers in deed. In order to bolster such a kind of systematic research, first of all, it is necessary to prepare capsaicinoids, firstly the capsaicin and dihydrocapsaicin, in sufficient high quality and in ample quantities as to undertake a systematic as well as multifactorial studies from different angles and points of view. To our best knowledge there are two principle routes, one is traditional by which the capsaicin and dihydrocapsaicin is separated and manufactured from the extracts extracted from the dry powders of hot peppers whose native texture contains capsaicin, dihydrocapsaicin, nordihydrocapsaicin, homocapsaicin, homodihydrocapsaicin etc., the other is artificial with the help of which individual capsaicinoid, such as capsaicin, dihydrocapsaicin can be synthesized by reacting vanillylamine and the corresponding acid chloride as outlined in Equation (1) of our previous article [1]. As far as the traditional route is concerned, it is advantageous and featured by the case of raw material available for massive production, the safety as drugs to be administrated to the patients and the plenty in supply. However anything in the world is twofold. The traditional route is likewise disadvantageous and characterized by a complexity of the technological production 
line, long term period of recycle and the extremely high price of the final products. In a word as compared with the traditional route, the artificial one had displayed a few distinguished superiorities, such as a comparatively shortened and reduced preparation and separation procedures and much lower production cost of the products.

Based on our experience and practice, all routes, whether they are traditional, artificial even or enzymatic as well [11], are required to have a liquid chromatographic analytical method able to separate and characterize the possible analogues of capsaicin or dihydrocapsaicin originated either from the natural raw materials in case of traditional procedures, or from the sub-standard chemical reagent raw material $(<95 \%$ purity , for example, isodecyl alcohol with minor impurities of iso-nonyl and iso-octyl alcohols, $<5 \%$ ) in case of artificial procedures for dihydrocapsaicin-making.

A survey and investigation of literature enables us to be aware of a fact that neither published papers nor approved patents can provide a method or a technology by which a good many (8 - 10) of capsaicinoids or artificial analogues of capsaicin and dihydrocapsaicin can be separated and characterized by 1 run of chromatographic test within a short period less than 40 minutes.

A required chromatographically analytical method comprising column, detector, fixed phase, flow phase etc. is addressed to separate the possible capsaicinoids such as capsaicin, dihydrocapsaicin, nordihydrocapsaicin, homocapsaicin and the artificial capsaicin analogues as listed in Table 1, at which is the right purpose of present study aimed, presented and discussed.

\section{Experiment}

\subsection{Analogues}

The chemical name, chemical formula of 8 synthesized analogues of capsaicin and dihydrocapsaicin in our laboratory according to the procedures outlined in paper [1] used for chromatographic separation and analysis are listed in Table 1.

\subsection{Analysis}

\subsubsection{Reagent}

Acetonitrile, chromatographic grade; Glacial acetic acid, 99.5\%, analytical grade; Ethanol, analytical grade manufactured by Handan Fine Chemical Company, Province Hebei, China.

\subsubsection{Sample Solution}

Approximately $0.016 \mathrm{~g}$ of each analogue sample is weighed precisely and dissolved in ethanol $(100 \mathrm{ml})$, from which an aliquot $(3 \mathrm{ml})$ was sucked out to form the analogues mixture stock solution. $20 \mu \mathrm{l}$ of the said stock solution, after passing through a filter membrane $(0.45 \mu$ pore diameter), was injected into the column used.

\subsubsection{Seeking for Optimum Separation Procedure Apparatus}

Liquid Chromatography Agileng 1200, Chromatographic Column A.E. Lichyon, C-18, $5 \mu \mathrm{m}$, two columns differ in length, one is $150 \mathrm{~mm}$, the other $250 \mathrm{~mm}$ respectively. Flow phase

1-st flow phase is methanol plus $\mathrm{H}_{2} \mathrm{O}$, whose relative percentage (\%) is prepared to be (methanol: $\mathrm{H}_{2} \mathrm{O}$ ) 50:50, 60:40, 65:35, 70:30 and 75:25.

The 2-nd flow phase is acetonitrile: $\mathrm{H}_{2} \mathrm{O}$ : glacial acetic acid whose percentage (\%) is consisted of 30:70:0.6, 40:60:0.6, 45:55:0.6 and 50:50:0.6 respectively.

A common normalized conditions (column C-18, 250 $\mathrm{mm}$ long, column temperature at $30^{\circ} \mathrm{C}$ and the constant flow rate $1.5 \mathrm{ml} / \mathrm{min}$ ) is used in an effort to seek for the optimum flow phase's experimental conditions beneficial to improve the separation results

\section{Results and Discussion}

\subsection{Optimization of Analytical Conditions}

Column

Prolongation of the column length from $150 \mathrm{~mm}$ to $250 \mathrm{~mm}$ has brought about an extention of 1 run from 42 minutes to 75 minutes, however created no remarkable improved separation efficiency, in particular for the analogues with much more closer molecular structures (No. 2, 3, 4 and 5 in Table 1). A shorter column $(150 \mathrm{~mm})$ is preferable for use.

Flow phase

A comparative study of a same solution but with different percentage (\%) proportions showed that the solution with the percentage proportion of ethanol: $\mathrm{H}_{2} \mathrm{O}$ $(75: 25)$ had a better promotion effect with regard to bettering a separation efficiency.

A similar promotion effect is also with the ternary system of acetonitrile: $\mathrm{H}_{2} \mathrm{O}$ : glacial acetic acid with percenttage proportion (45:55:0.6). In order to save space all the 9 chromatogram maps for above 9 proportions (\%) are omitted.

Elution rate

An experimental attempt was made to watch in which degree an deviation from an usual elution rate of 1.5 $\mathrm{ml} / \mathrm{min}$ can be affected. Neither a deviation change from $1.5 \mathrm{ml} / \mathrm{min}$ to a smaller rate, such as $0.85 \mathrm{ml} / \mathrm{min}$, nor to a longer rate, for example, to $2.0 \mathrm{ml} / \mathrm{min}$ could brought about a bit improvement. A middle rate is the best of 1.5 $\mathrm{ml} / \mathrm{min}$.

From the above-mentioned comparative studies it permits to make a fundamental conclusion that an optimum experimental conditions for chromatographic separation 
Table 1. The structure, retention time (RT), peak area and percentage content (\%) of capsaicinoids and analogues.

\begin{tabular}{|c|c|c|c|c|c|}
\hline No. & Structure & $\mathrm{RT}_{\mathrm{s}}$ & $\mathrm{RT}_{\mathrm{m}}$ & $A_{x}$ & $\%$ \\
\hline 1 & & 14.34 & 14.25 & 198.4 & 11.24 \\
\hline 2 & & 22.01 & 21.87 & 39.8 & 2.26 \\
\hline 3 & & 12.97 & 12.91 & 75.46 & 4.28 \\
\hline 4 & & 11.20 & 11.15 & 734.3 & 41.60 \\
\hline 5 & & 15.06 & 14.99 & 130.8 & 7.41 \\
\hline 6 & & 38.28 & 38.50 & 99.8 & 5.66 \\
\hline 7 & & 13.67 & 13.59 & 237.7 & 13.47 \\
\hline 8 & & 16.75 & 16.68 & 249.2 & 14.12 \\
\hline
\end{tabular}

$\mathrm{RT}_{\mathrm{s}}$ : retention time for single sample injection; $\mathrm{RT}_{\mathrm{m}}$ : for mixture sample injection.

of analogues of capsaicin and (or) dihydrocapsaicin can be recommended as follows: column $\mathrm{C}-18,150 \times 4.6$ $\mathrm{mm}, 5 \mu \mathrm{m}$, flow phase acetonitrile- $\mathrm{H}_{2} \mathrm{O}$-glacial acetic acid in percentage proportion 45.0:54.4:0.6; flow rate of eluent $1.5 \mathrm{ml} / \mathrm{min}$ and a temperature of column $30^{\circ} \mathrm{C}$.

Under the optimized conditions each individual analogues's solution $(20 \mu \mathrm{l})$ was injected alone and the specific retention time for quantitative analysis was found. The experimentally found retention time for all the 8 analogues was determined and summarized in the figure note of Figure 1. From Figure 1 it can be seen that the retention time of each individual analogue obtained by single analogue injection (No. 1-8) are in very good coincidence with that found by mixture analogue ingection within an allowed deviations and that among the 8 analogues (No. 1-8 in Table 1) 7 analogues are separated and characterized quite well with an unique fault that showed an overlapping of the retention time of No. 7with 


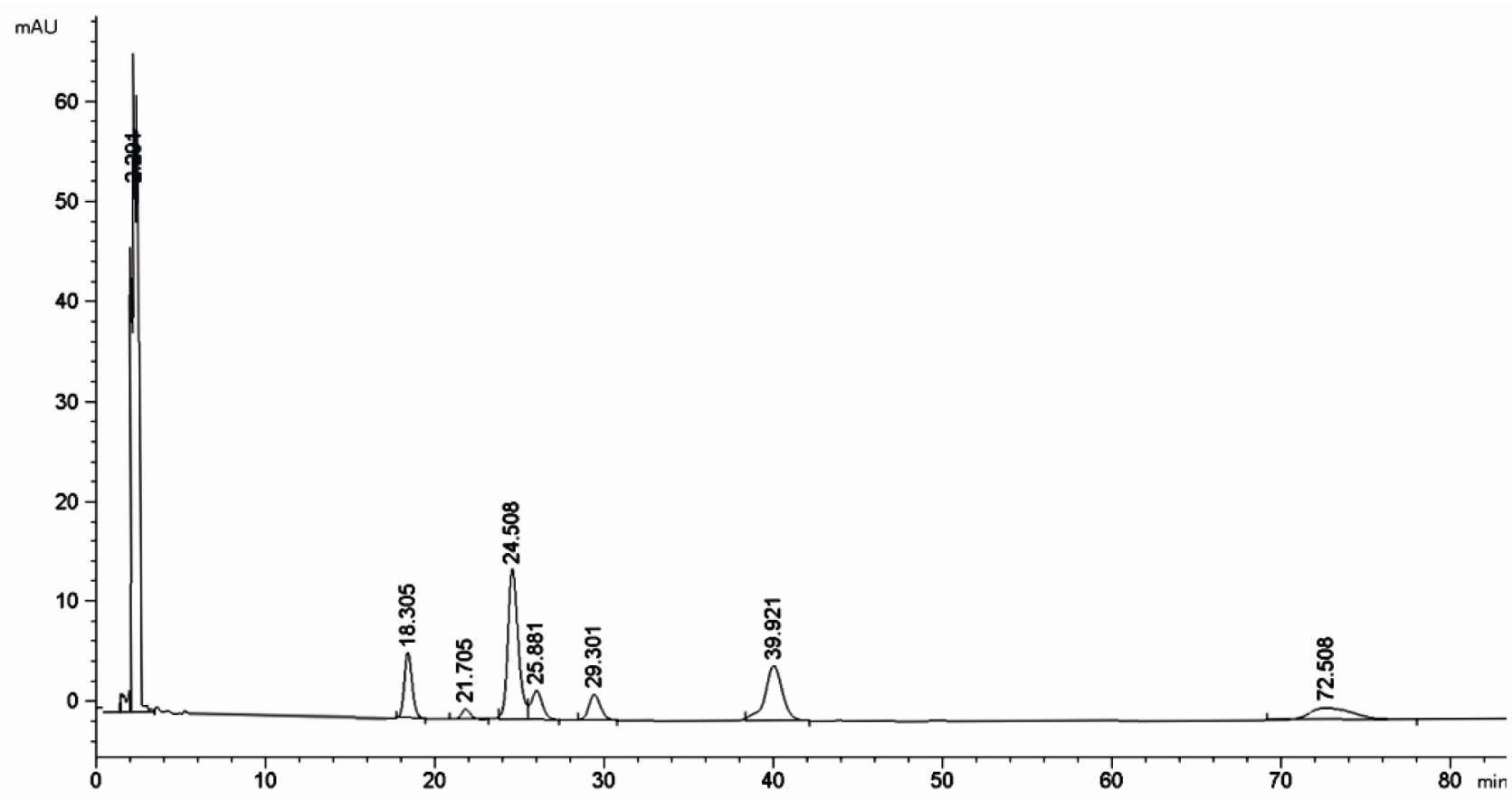

Figure 1. Chromatogram map for separation of No. 1-5 and No. 7-8.

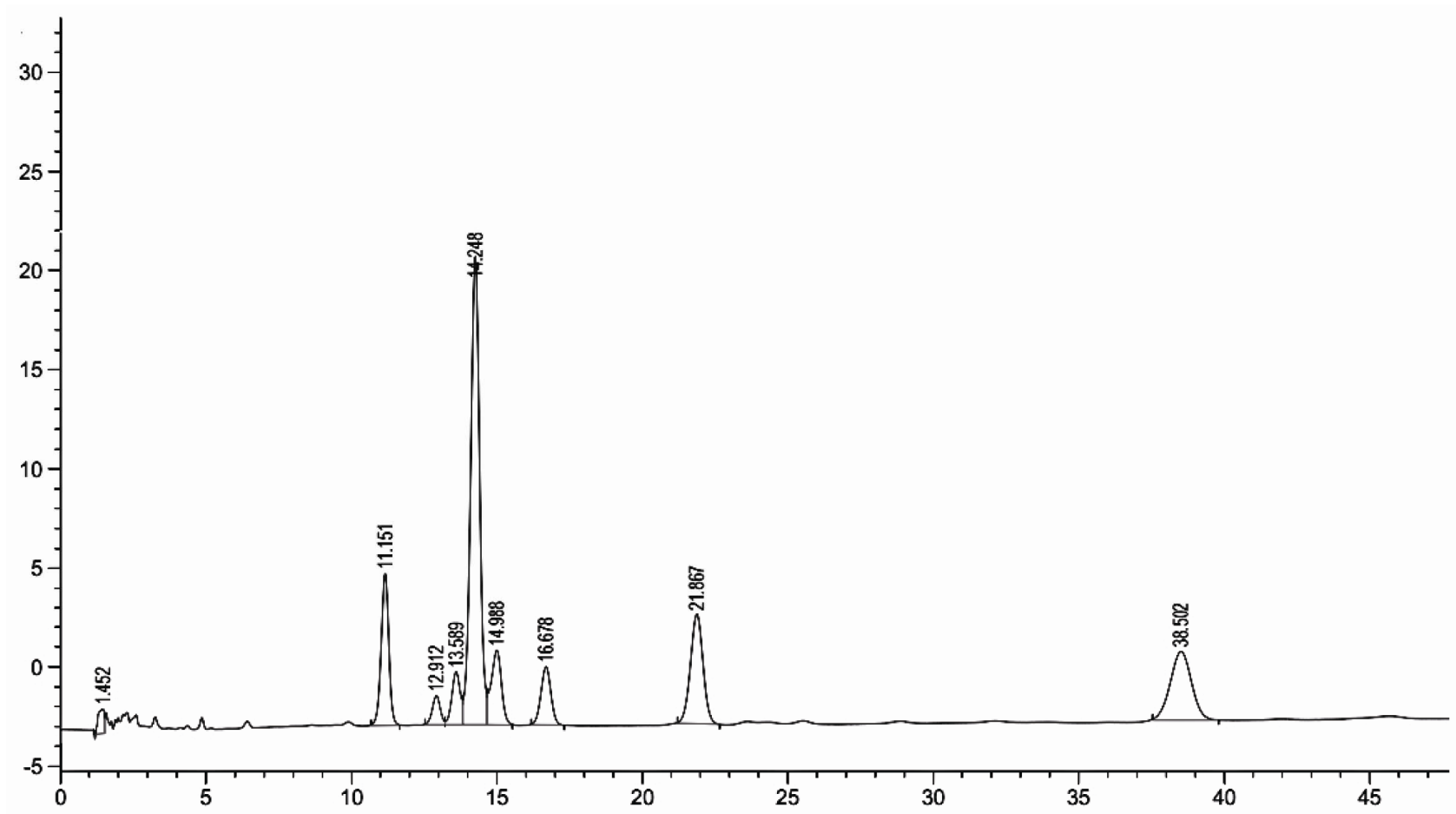

Figure 2. Chromatogram map for separation of No. 1-8.

that of No. 6. In order to avoid and overcome this fault a fine tuning of the acidity of the ternary eluent system was made and the 8 analogues' mixture was basically separated and quantitatively determined without over- lapping peaks like No. 6 and No. 7 (Figure 2).

The establishment of chromatographic separation and characterization is of practical significance. Firstly for analysis of natural capsaicinoids, in principle, applied 
with minor modification, the analyzing scope of capsaicinoids may be expanded to the minor members of capsaicinoid, such as homohomocapsaicin and nornordihydrocapsaicin etc. whose content in native hot pepper amount totally less than $2 \%-3 \%$; Secondly for analysis of artificial analogues including those pungent compounds with similar or approximate structures to each other that are not existed in native hot pepper but are able to be synthesized in chemical laboratory. It is believed that the recommended chromatographic procedure may promote the preparative progress of high-qualified capsaicinoids and their analogues that hold promise of being candidate drugs for a potential anticarcinogenic use.

\subsection{Summary}

1) An optimized Chromatographically operational conditions for a apsaicinoids' mixture separation can be summarized as follows: column C-18, $250 \times 4.6 \mathrm{~mm}$. 5u, flow phase acetonitrile $-\mathrm{H}_{2} \mathrm{O}$ - glacial acetic acid in percentage (V/V) 45.0; 54.4:0.6, flow rate of eluent 1.5 $\mathrm{ml} / \mathrm{min}$ and a column temperature at $30^{\circ} \mathrm{C}$.

2) 8 capsaicinoids/analogues are able to be separated and characterized with an allowed deviations and within a short period of 36 munites via 1 chromatographic experiment.

3) The suggested chromatographic analytical method holds promise to be used in principle for separating and characterizing native capsaicinoids as well as artificial analogues for medical usage.

\section{References}

[1] J. L. Wang, Z. H. Peng, X. B. Zhang and B. X. Peng, "A Study of Pungency of Capsaicinoids as Affected by Their Molecular Structure Alteration," Pharmacology and Pharmacy, in Press.

[2] A. Mori, et al., "Capsaicin, a Component of Red Peppers, Inhibits the Growth of Androgen-Independent, p53 Mutant Prostate Cancer Cells," Cancer Research, Vol. 66, No. 6, 2006, pp. 3222-3229. doi:10.1158/0008-5472.CAN-05-0087
[3] D. J. Morre, et al., "NADH Oxidase Activity from Sera Altered by Capsaicin Is Widely Distributed among Cancer Patients," Archives of Biochemistry and Biophysics, Vol. 342, No. 2, 1997, pp. 224-230. doi:10.1006/abbi.1997.0110

[4] A. M. Sanchez, et al., "Induction of the Endoplasmic Reticulum Stress Protein GADD153/CHOP by Capsaicin in Prostate PC-3 Cells: A Microarray Study," Biochemical and Biophysical Research Communications, Vol. 372, No. 4, 2008, pp. 785-791. doi:10.1016/j.bbrc.2008.05.138

[5] S. Malagarie-Cazenave, et al., "Capsaicin, a Component of Red Peppers, Induces Expression of Androgen Receptor via PI3K and MAPK Pathways in Prostate LNCaP cells," FEBS Letters, Vol. 583, No. 1, 2009, pp. 141-147. doi:10.1016/j.febslet.2008.11.038

[6] A. M. Sanchez, et al., "Apoptosis Induced by Capsaicin in Prostate PC-3 Cells Involves Ceramide Accumulation, Neutral Sphingomyelinase, and JNK Activation," Apoptosis, Vol. 12, No. 11, 2007, pp. 2013-2024. doi:10.1007/s10495-007-0119-z

[7] K. C. Brown, et al., "Capsaicin Displays Anti-Proliferative Activity against Human Small Cell Lung Cancer in Cell Culture and Nude Mice Models via the E2F Pathway," Plos One, Vol. 5, No. 4, 2010.

[8] K. Ito, et al., "Induction of Apoptosis in Leukemic Cells by Homovanillic Acid Derivative, Capsaicin, through Oxidative Stress: Implication of Phosphorylation of p53 at Ser-15 Residue by Reactive Oxygen Species," Cancer Research, Vol. 64, No. 3, 2004, pp. 1071-1078. doi:10.1158/0008-5472.CAN-03-1670

[9] G. Galati and P. J. O'Brien, "Cytoprotective and Anticancer Properties of Coenzyme Q versus Capsaicin," Biofactors, Vol. 18, No. 1-4, 2003, pp. 195-205. doi:10.1002/biof.5520180222

[10] I. Diaz-Laviada, "Effect of Capsaicin on Prostate Cancer Cells," Future Oncology, Vol. 6, No. 10, 2010, pp. 1545 1550. doi: $10.2217 /$ fon. 10.117

[11] K. Kobata, et al., "Enzymatic Synthesis of Capsaicin Analogs with Liver Acetone Powder," Tetrahedron Letters, Vol. 37, No. 16, 1996, pp. 2789-2790. doi:10.1016/0040-4039(96)00429-7 